\title{
Poor Prognosis of Pulmonary Adenosquamous Carcinoma with NRAS and HRAS Double Mutation
}

This article was published in the following Dove Press journal: OncoTargets and Therapy

\author{
Jidong Zhao' \\ Xiangmei Zhang ${ }^{2}$ \\ Ming $\mathrm{He}^{\text {' }}$ \\ Xin Chen ${ }^{\prime}$ \\ Xing Cui' \\ Tian Qin ${ }^{3}$ \\ Xueliang $\mathrm{Niu}^{3}$ \\ Liyan Zhao ${ }^{4}$
}

'Department of Thoracic Surgery, The Fourth Hospital of Hebei Medical University, Shijiazhuang, People's Republic of China; ${ }^{2}$ Research Center, The Fourth Hospital of Hebei Medical University, Shijiazhuang, People's Republic of China; ${ }^{3}$ Medical Department, Burning Rock Biotech, Guangzhou, People's Republic of China; ${ }^{4}$ Department of Internal Medicine, The First Hospital of Xingtai, Xingtai, People's Republic of China
Correspondence: Xin Chen

Department of Thoracic Surgery, The

Fourth Hospital of Hebei Medical

University, No. 12, Jiankang Road,

Shijiazhuang, Hebei Province, 0500I0,

People's Republic of China

Tel +86-13633II8699

Email 2029669284@qq.com

\begin{abstract}
RAS mutations constitute one of the major tumorigenic mechanisms and are detected in approximately $20 \%$ of lung cancers. The most frequent mutated and well-studied RAS isoform is KRAS, which is associated with an overall poor prognosis in non-small-cell lung cancer (NSCLC). However, the clinical significances of NRAS and HRAS in NSCLC are rarely reported. Here, we present a 58-year-old male smoker who was diagnosed with stage IV lung adenosquamous carcinoma. A rare NRAS and $H R A S$ double mutation was detected in the primary tumor and lymph node samples using next-generation sequencing (NGS). The patient showed rapid disease progression and passed away due to respiratory failure after 15 days of osimertinib in combination with cisplatin. To the best of our knowledge, this is the first report associating NRAS and HRAS double mutation in the poor prognosis of NSCLC.
\end{abstract} Keywords: NSCLC, NRAS, HRAS

\section{Introduction}

The RAS oncogene affects numerous cellular functions, including proliferation, growth, apoptosis, migration, division, and differentiation of the cells. It has 3 known isoforms: Harvey-RAS (HRAS), Kirsten-RAS (KRAS), and neuroblastomaRAS (NRAS). ${ }^{1}$ The incidence of $K R A S$ mutation in lung adenocarcinoma is between $12 \%$ and $36 \%$. Previous studies have demonstrated that KRAS mutation frequently occurs in lung adenocarcinoma patients who are former or current smokers and are associated with poor overall prognosis. ${ }^{2,3}$ In contrast, NRAS and $H R A S$ mutations are extremely rare in lung cancer regardless of histopathological subtypes, with only a few cases reported in the literature. Here, we present a case of pulmonary adenosquamous carcinoma harboring $N R A S$ and $H R A S$ double mutation, who failed to respond to chemotherapy plus targeted therapy.

\section{Case Presentation}

A 58-year-old male smoker presented to our hospital with one-month history of chest and back pain. The performance status (PS) value of the patient was 0 . Systemic evaluation including brain magnetic resonance imaging (MRI) and contrast-enhanced computed tomography (CT) scan showed a cavitary nodule on the inferior lobe of the right lung and the presence of malignant pleural effusion (Figure 1). Histopathology review of the tissue biopsy sample suggested stage IV poorly differentiated carcinoma. Twelve days after admission, malignant pleural effusion was aggravated and a video-assisted thoracoscopic lobectomy and lymph node dissection was performed for symptom management. Pathological examination of 

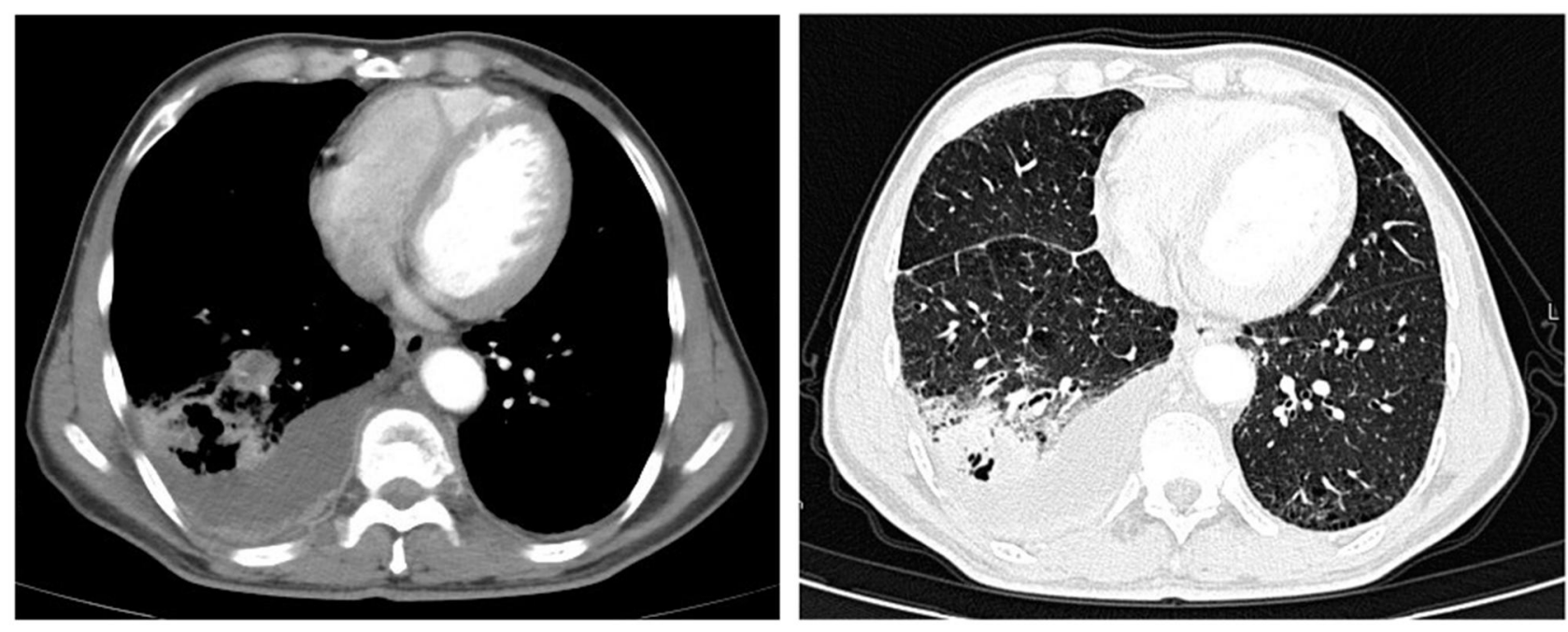

Figure I Chest CT at the time of the diagnosis.

tumor, visceral pleura, and lymph nodes confirmed the diagnosis of poorly differentiated adenosquamous carcinoma and the presence of tumor cells in the visceral pleura and lymph nodes. Amplification refractory mutation system (ARMS)-polymerase chain reaction (PCR) assay was performed using pleural effusion samples to profile the EGFR mutation status, which showed weak signals for L858R and T790M mutations (CT value: 43.83 and 40.67 , respectively, Figure 2A). The patient was administered with third-generation EGFR-TKI osimertinib (150 mg, orally thrice daily) combined with cisplatin (40 mg every ten days). After 5 days of osimertinib treatment, pleural effusion was reduced. Unfortunately, the patient showed a rapid disease progression and died of respiratory failure after 15 days of osimertinib plus cisplatin administration. To understand the underlying mechanism of treatment failure, both surgical and lymph node tissue samples were subjected to capture-based ultra-deep sequencing that target the exons and critical introns of 520 cancer-related genes (Oncoscreen Plus ${ }^{\mathrm{TM}}$, Burning Rock Biotech, China). HRAS Q61L and NRAS Q61K were consistently found in the primary tumor and lymph nodes (Figure 2B and C). However, EGFR mutations were not detected, indicating that the EGFR L858R and T790M detected by ARMS-PCR may be false-positive mutations.

\section{Discussion}

This case reported the poor prognosis of pulmonary adenosquamous carcinoma with $N R A S$ and $H R A S$ mutation. The patient had a rapid progression leading to death. To the best of our knowledge, this is the first case which describes the detection of $N R A S / H R A S$ double mutation and its prognostic value in lung cancer.

RAS mutations constitute a major tumorigenic mechanism and are detectable in approximately $20 \%$ of lung cancers. The most common KRAS mutations account for $80-90 \%$ of all $R A S$ mutations. KRAS mutations are generally considered to be a negative predictive factor for EGFR-tyrosine kinase inhibitor (TKI) therapy and are associated with a poor overall prognosis in NSCLC., ${ }^{2,3}$ $H R A S$ and NRAS mutations have been reported in various cancer types including head and neck carcinoma, gastrointestinal cancer, genitourinary cancer, and malignant melanoma. ${ }^{4-7}$ The frequency of HRAS and NRAS mutations in bladder cancer is up to $84 \% .{ }^{8}$ In colorectal cancer, patients with any known KRAS or NRAS mutation should not be treated with either cetuximab or panitumumab according to the NCCN guidelines. Likewise, HRASmutated colorectal cancer also results in the resistance to anti-EGFR therapies. ${ }^{9}$ However, HRAS and NRAS mutations are extremely rare in lung cancer; hence, studies documenting their clinical significance are scarce. Cathcart-Rake et al have reported an elderly former smoker with HRAS mutant NSCLC. The patient had a rapid progression from stage IB disease to metastatic adenocarcinoma and death, indicating that HRAS mutations are associated with poor overall prognosis in NSCLC, similar to $K R A S .{ }^{10}$ An in vitro study has showed that mutant $H R A S$ hyperactivates the RAS and the mTOR pathway in various cancer cell lines including lung cancer, which might serve as a therapeutic target. ${ }^{11}$ NRAS mutations are more common in current/former smokers with NSCLC 

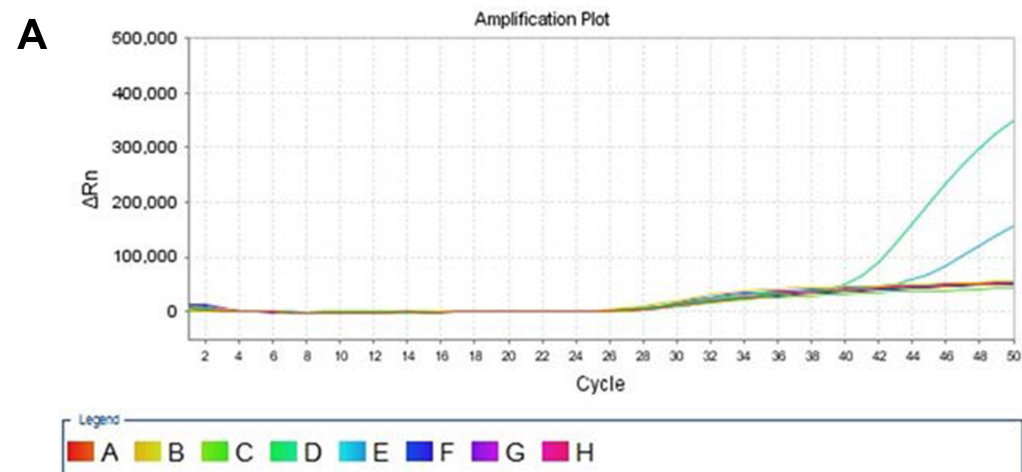

B
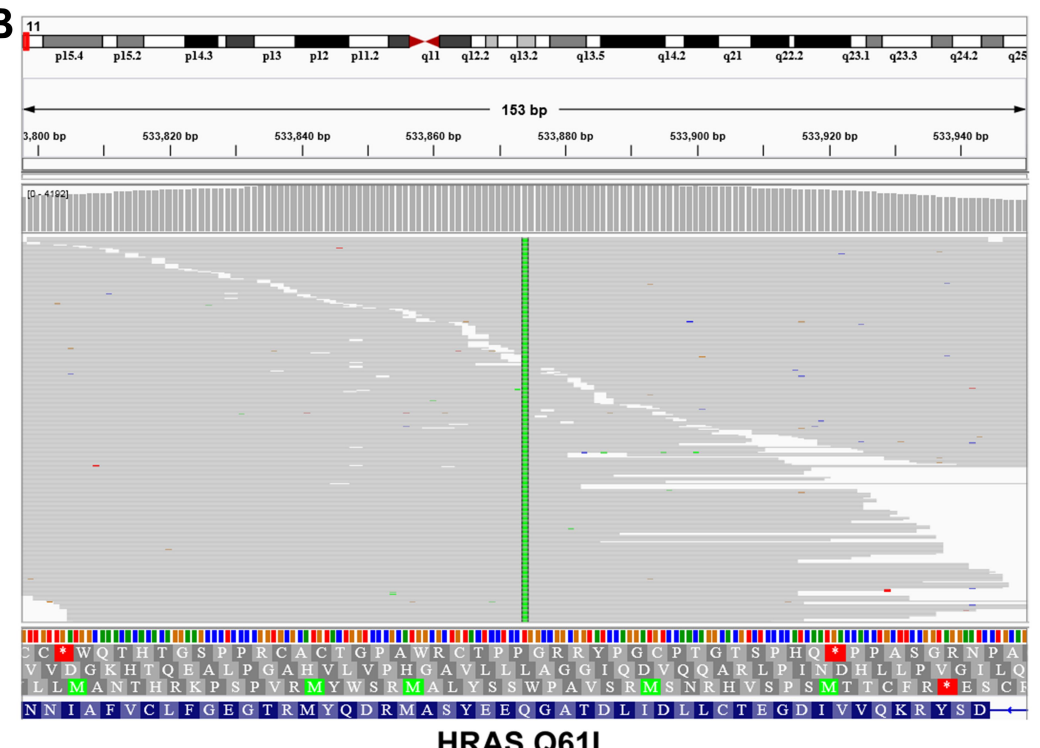

HRAS Q61L
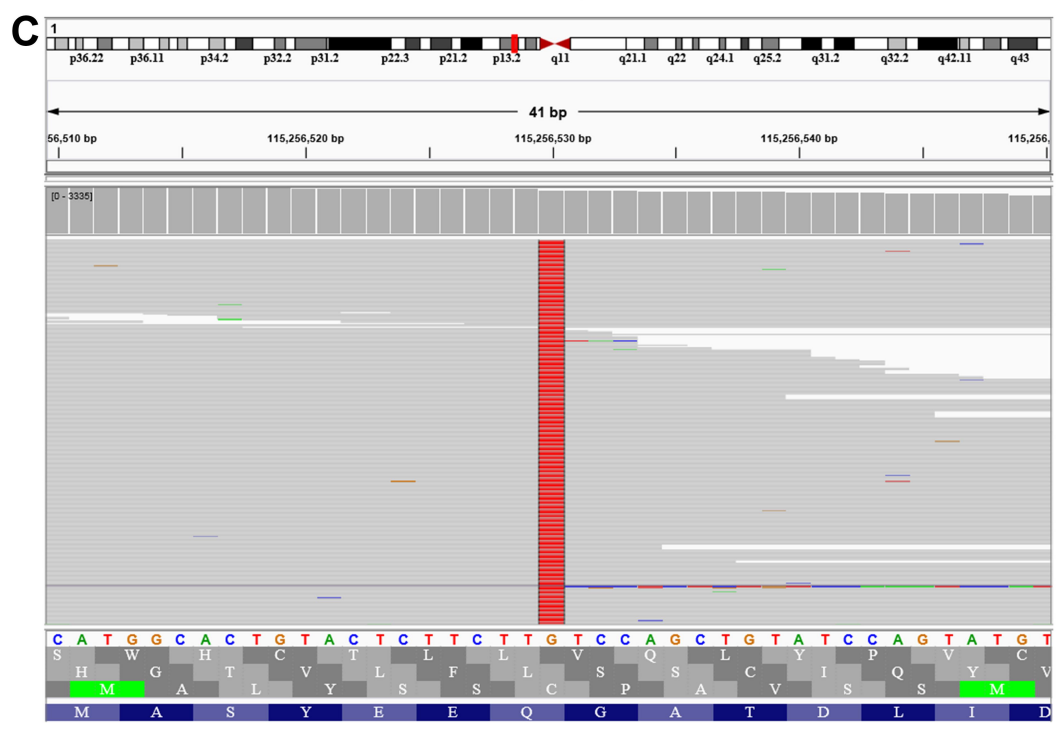

NRAS Q61K

Figure 2 ARMS-PCR demonstrated EGFR mutations L858R and T790M (A) and NGS identified HRAS Q6IL (B) and NRAS Q6IK (C) mutations. 
and NRAS-mutant cell lines were demonstrated to be sensitive to the MEK inhibitors, selumetinib and trametinib. ${ }^{12}$

While the single mutation in either $N R A S$ or $H R A S$ is rare in lung cancer, the double mutation in $N R A S$ and $H R A S$, which we present in this case report, is even rarer and had never been reported before. The patient had rapid progression and died about 1 month after diagnosis. It is worth mentioning that EGFR L858R/T790M mutations were detected by ARMS-PCR in the pleural effusion sample, prompting the administration of osimertinib. However, no EGFR mutation was found by NGS, a more precise and comprehensive methodology for mutation analysis. Whether tumor heterogeneity or false detection that brought about these inconsistent results from the two molecular assays is unclear; however, the use of EGFRTKIs could partly account for the patient's poor prognosis. In conclusion, our case indicates that NRAS and HRAS mutations in NSCLC are associated with poor prognosis. Further research and description of clinical cases are needed for improved better understanding of these genetic mutations in NSCLC.

\section{Ethical Approval}

Institutional approval was not required to publish the case details.

\section{Patient Informed Consent}

Written informed consent was obtained from the patient for the publication of his case details and images.

\section{Acknowledgments}

We are grateful to the patient and her family. Also, we wish to express our gratitude to the clinical investigators, study coordinators, operation staff, and all other team members on this project.

\section{Disclosure}

Tian Qin and Xueliang Niu are affiliated with Burning Rock Biotech. The authors declare no other conflicts of interest.

\section{References}

1. Cox AD, Fesik SW, Kimmelman AC, Luo J, Der CJ. Drugging the undruggable RAS: mission possible? Nat Rev Drug Discov. 2014;13 (11):828-851.

2. Karachaliou N, Mayo C, Costa C, et al. KRAS mutations in lung cancer. Clin Lung Cancer. 2013;14(3):205-214. doi:10.1016/j. cllc.2012.09.007

3. Chapman AM, Sun KY, Ruestow P, Cowan DM, Madl AK. Lung cancer mutation profile of EGFR, ALK, and KRAS: meta-analysis and comparison of never and ever smokers. Lung Cancer. 2016;102:122-134. doi:10.1016/j.lungcan.2016.10.010

4. Clinkscales W, Ong A, Nguyen S, Harruff EE, Gillespie MB. Diagnostic value of RAS mutations in indeterminate thyroid nodules. Otolaryngol Head Neck Surg. 2017;156(3):472-479. doi: $10.1177 / 0194599816685697$

5. Cicenas J, Tamosaitis L, Kvederaviciute K, et al. KRAS, NRAS and BRAF mutations in colorectal cancer and melanoma. Med Oncol. 2017;34(2):26. doi:10.1007/s12032-016-0879-9

6. Tripathi K, Goel A, Singhai A, Garg M. Mutational analysis of Ras hotspots in patients with urothelial carcinoma of the bladder. World J Clin Oncol. 2020;11(8):614-628. doi:10.5306/wjco.v11.i8.614

7. Nassar KW, Tan AC. The mutational landscape of mucosal melanoma. Semin Cancer Biol. 2020;61:139-148. doi:10.1016/j. semcancer.2019.09.013

8. Mo L, Zheng X, Huang HY, et al. Hyperactivation of Ha-ras oncogene, but not Ink4a/Arf deficiency, triggers bladder tumorigenesis. J Clin Invest. 2007;117(2):314-325. doi:10.1172/JCI30062

9. Boidot R, Chevrier S, Julie V, Ladoire S, Ghiringhelli F. HRAS G13D, a new mutation implicated in the resistance to anti-EGFR therapies in colorectal cancer, a case report. Int J Colorectal Dis. 2016;31(6):1245-1246. doi:10.1007/s00384-015-2448-7

10. Cathcart-Rake E, Corless C, Sauer D, Lopez-Chavez A. Elderly former smoker with HRAS mutant non-small-cell lung cancer. $J \quad$ Thorac Oncol. 2014;9(10):e75-78. doi:10.1097/ JTO.0000000000000315

11. Kiessling MK, Curioni-Fontecedro A, Samaras P, et al. Mutant HRAS as novel target for MEK and mTOR inhibitors. Oncotarget. 2015;6(39):42183-42196. doi:10.18632/oncotarget.5619

12. Ohashi K, Sequist LV, Arcila ME, et al. Characteristics of lung cancers harboring NRAS mutations. Clin Cancer Res. 2013;19 (9):2584-2591. doi:10.1158/1078-0432.CCR-12-3173
OncoTargets and Therapy

\section{Publish your work in this journal}

OncoTargets and Therapy is an international, peer-reviewed, open access journal focusing on the pathological basis of all cancers, potential targets for therapy and treatment protocols employed to improve the management of cancer patients. The journal also focuses on the impact of management programs and new therapeutic agents and protocols on patient perspectives such as quality of life, adherence and satisfaction. The manuscript management system is completely online and includes a very quick and fair peer-review system, which is all easy to use. Visit http://www.dovepress.com/ testimonials.php to read real quotes from published authors. 